\title{
Improvement of two-dimensional gel electrophoresis conditions for rin mutant tomato fruit
}

$$
\begin{aligned}
& \text { Ling } \mathrm{Li}^{1,2, a} \text {, Yuanyuan Song }{ }^{1, b} \text {, Yifeng Wang }{ }^{1, c} \text {, Baoxin Zheng }{ }^{1, d} \text { and Tieling } \\
& \operatorname{Liu}^{1,2, e, ~ *}
\end{aligned}
$$

${ }^{1}$ College of Food Science and Biotechnology, Tianjin Agricultural University, Tianjin 300384, China

${ }^{2}$ Tianjin Engineering and Technology Research Center of Agricultural Products Processing, Tianjin 300384, China

aliling19820925@163.com, 'bongyuan19931016@163.com, 'poi987246@163.com, dzhengshipin3933@163.com, ${ }^{\mathrm{e}} \mathrm{t} \mid \mathrm{@tjau.edu.cn}$

*To whom correspondence should be addressed.

Key words: mutant rin, proteomics, two-dimensional gel electrophoresis, optimum condition Abstract. Tomato fruit contains a lot of pigment, polysaccharide and secondary metabolism substances. These substances can affect protein separation effect, and interfere with the process of two-dimensional gel electrophoresis. The extraction of tomato fruit protein sample, conditions of isoelectric focusing and two-dimensional gel electrophoresis had been investigated to establish a suitable method of rin mutant tomato fruit. It was shown that the TCA-acetone precipitation combined with clean-up kit was the best method for tomato fruit protein extraction. The best condition was $\mathrm{pH}$ 4-7 IPG, lysis buffer II, $500 \mu \mathrm{g}$ sample. The 2-DE protocol suited for the separation of proteins from rin mutant tomato fruit was established.

\section{Introduction}

The two-dimensional gel electrophoresis (2-DE) has the characteristics of high resolution and information, and is a powerful tool for proteomics. It has been widely used in plants, animals, microorganisms and other fields [1,2]. Especially, with the maturity and perfection of the technology of mass spectrometry analysis, the 2-DE-MS, is the core technology of proteomics, had speedily developed. Plant cells contain many secondary metabolites, which have important effects on the proteins extraction, separation and purification [3]. At the same time, the extraction efficiency of some low concentrations protein and insoluble protein is low. In order to improve the resolution of the protein, the IPG gel strip was generally used to make the protein samples separation according to different $\mathrm{pH}$.

Tomato is an important vegetable, raw material for food industry, and has great economic value. Tomato is also a very good model plant, which has been a hot research in proteomics. Vrebalov et al [4] obtained the rin mutan, found rin deletion about 3kb, which resulted in the two MADS-box genes (LeMADS-RIN and LeMADS-MC) loss biological activity and could not transmit signal to the ethylene receptor [5]. The function of gene is realized by encoding protein. Protein is the embodiment and the executor of biological activity [6]. Therefore, in order to study the tomato fruit from the protein level, the optimal experimental for the preparation method of total protein, electric focusing and two-dimensional electrophoresis were must first determined. The purpose of this study is to construct the optimal conditions for the two-dimensional electrophoresis of rin tomato fruit 
and provide the theoretical basis and technical support for the research of tomato fruit proteomics.

\section{Materials and Methods}

Plant materials. Tomato (Lycopersicon esculentum Mill. cv. rin mutant) plants were grown in a greenhouse $\left(25{ }^{\circ} \mathrm{C} / 20{ }^{\circ} \mathrm{C}\right.$ and $90 \%$ humidity under $14 \mathrm{~h} / 10 \mathrm{~h}$, light/dark) at Ji County of Tianjin. Fruits were harvested at breaker stage and selected free from visual defects and for uniformity of weight and shape.

Protein extraction and quantitative determination. The Protein of tomato samples were extracted by TCA-acetone method. After that, the samples were treated by clean-up kit according to protocol. Finally, protein samples were quantified by 2-D Quant-kit. In this experiment, the standard curve is $\mathrm{y}=5.1501 \mathrm{x}+0.0206\left(\mathrm{R}^{2}=0.991\right)$.

Protein isoelectric focusing. The $300 \mu \mathrm{g}$ and $500 \mu \mathrm{g}$ protein samples, $0.8 \%$ IPG buffer were respectively added in centrifugal tube, and the homogenate was centrifuged at 20,000 g for $10 \mathrm{~min}$. Then, the protective layer of IPG strip was gently removed, and the IPG strip were placed on the sample solution. Isoelectric focusing was performed using an Ettan IPGphor unit under the following conditions: $50 \mathrm{~V}, 500 \mathrm{~V}$ and $1000 \mathrm{~V}$ for $1 \mathrm{~h}$, respectively; $4000 \mathrm{~V}$ and $8000 \mathrm{~V}$ for $2 \mathrm{~h}$ and $8000 \mathrm{~V}$ until final volt-hours $(80 \mathrm{kV} \mathrm{h})$. The balance and second SDS-PAGE were immediately performed at the end of isoelectric focusing.

Two-dimensional gel electrophoresis of total protein extracts. The 2-DE was performed using $13 \%$ polyacrylamide gels. Electrophoresis was carried out at $20^{\circ} \mathrm{C}$ and $1.0 \mathrm{~W} / \mathrm{gel}$ for $2 \mathrm{~h}$, and at 15 $\mathrm{W} / \mathrm{gel}$ until the dye front reached about $1 \mathrm{~cm}$ from the bottom of the gel. After electrophoresis, the gels were stained with Coomassie brilliant blue (CBB) R-250. To minimize errors, the proteomic analysis at each treatment was conducted in triplicate. The CBB-stained gels were scanned by a UMAX Powerlook 2100XL-USB scanner and stored in TIF format.

\section{Results and Discussion}

Extraction of total protein. In order to attain high-quality and good repeatability 2-DE profile, extraction of total protein is one of the most basic and important steps [7]. Tomato fruit contains a lot of pigment, polysaccharide and other secondary metabolites, which can affect the separation effect of protein and interfere with 2-DE, and easily cause the horizontal stripes in profiles [8].

This study used TCA-acetone precipitation to extract rin tomato fruit protein. As shown in Fig.1A, extracted protein mainly focued on 14.4KD-94KD. There were many transverse stripes, tailing phenomenon, and low resolution in the Fig.1A. We next used clean-up kit to deal with the extracted sample (Fig.1B). In the Fig.1B, transverse stripes reduced, effective proteins increased and the level of tailing phenomenon dropped. Therefore, on the basis of TCA-acetone precipitation, using clean-up kit to deal with the extracted sample, which could get the satisfactory 2-DE profiles. The result was in accordance with Wang et al [9].

Determination of lysis buffer system. The tomato fruit protein samples dissolved absolutely which was in favor of confirming the appropriate isoelectric focusing condition, so lysis buffer should make the protein sample reached the maximal solubility. Lysis bufferlcontained $8 \mathrm{M}$ urea,

4\% (w/v) CHAPS, 2M thiourea, 20mM DTT, 2\% Pharmalyte4-7. Lysis bufferllincluded 9M Urea, 4\% (w/v) CHAPS, 0.5\% TritonX-100, 20mM DTT, 2\% Pharmalyte4-7. The tomato fruit protein 
respectively dissociate via Lysis bufferland Lysis bufferll, then two dimensional electrophoresis were conducted (Fig.2A, 2B). It was indicated that protein points were clear and round in Fig.2B, and were more than Fig.2A. The overall effect of the Fig.2B was better than Fig.2A.

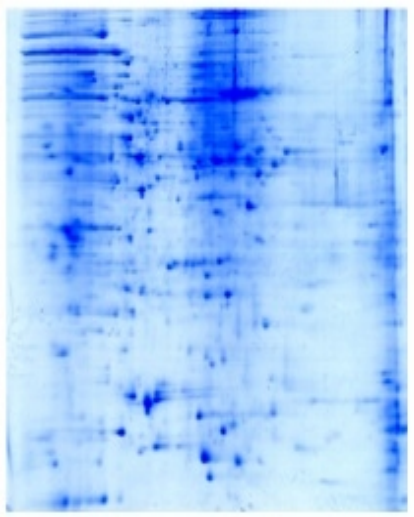

A

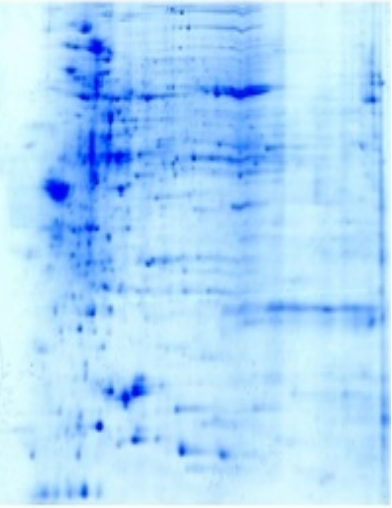

B

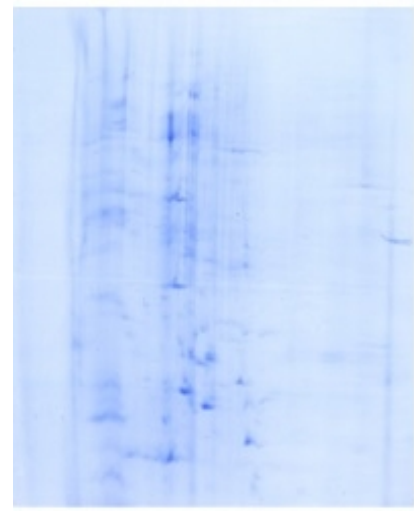

$\mathbf{A}$

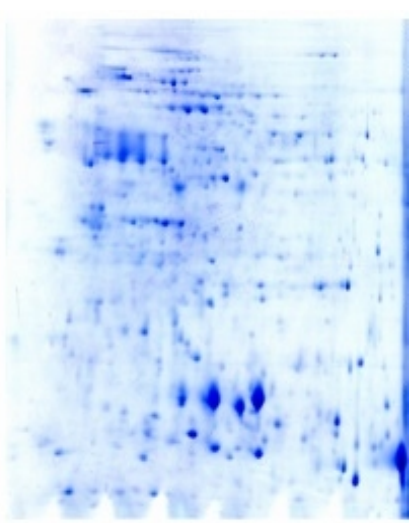

B

Fig 1

Fig 2

Fig.1 2-DE profile of different total protein extraction methods on tomato

Fig.2 2-DE profile of different lysis buffers

Determination of sample quantity in 2-DE. The amount of protein is also an important factor affecting the results of 2-DE. TCA-acetone precipitation and clean-up kit, pH4-7 and 18cm IPG trip were used in this experiment. In order to determine the optimal sample quantity for the 2-DE of rin mutant tomato fruit, $300 \mu \mathrm{g}$ and $500 \mu \mathrm{g}$ protein was added in 2-DE. It was shown that when the sample quantity was $300 \mu \mathrm{g}$, the number of protein spots in 2-DE map was less and very fuzzy, and the resolution was lower (Fig.3A), while when the sample quantity was $500 \mu \mathrm{g}$ (Fig.3B), the number of protein spots were significantly increased, and the protein spots were clearly visible. Together, the resolution and the quality of protein spots in Fig.3A were higher than that of in Fig.3B.

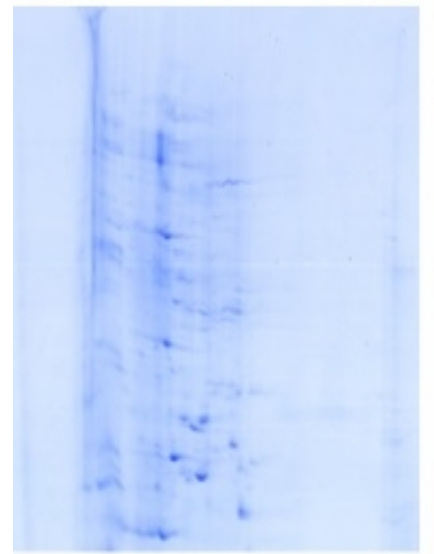

A

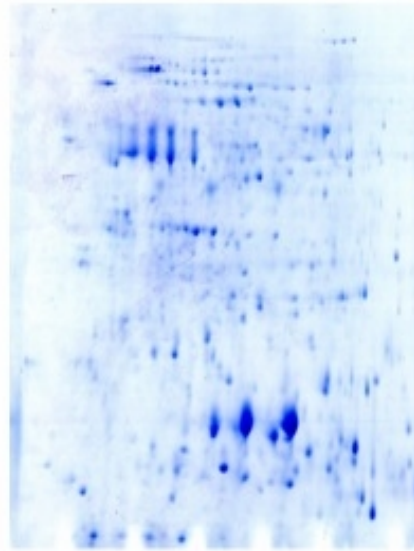

B

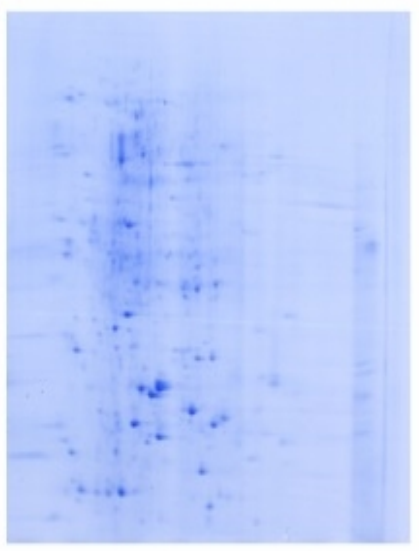

A

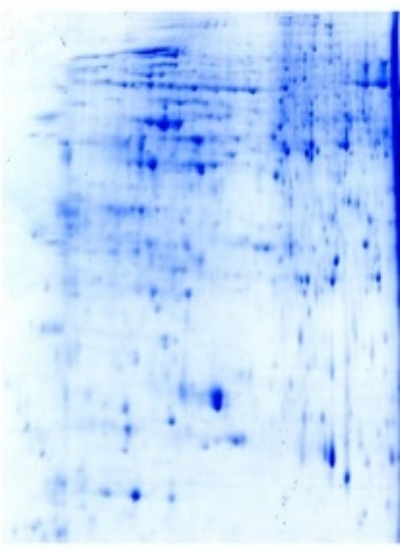

B

Fig. 3

Fig. 4

Fig.3 2-DE profile of different loaded samples

Fig.4 2-DE profile of different $\mathrm{pH}$ 
Selection of optimal pH conditions. To choose the most suitable pH of IPG strip for detaching proteins of tomato fruit, compare the detaching effects of the IPG strip which the pH was 3-10 (Fig. 4A) and 4-7 (Fig.4B). Use the ImageMaster 2D Platinum 5.0 software to analysis the profiles, it was found that the number of protein points in Fig.4A was 438, and was 663 in Fig.4B. The results suggested that the $\mathrm{pH} 4-7$ of IPG strip increased the quantity of protein points by detaching and distinguish ability, as well as the appearance of low abundance proteins. The result was in accordance with Lu et al [10].

The profiles of rin mutant tomato fruit under the best condition. The profiles of rin mutant tomato fruit under the best condition was shown in Fig.5. It was shown that protein points evenly distributed in the whole profile, were relatively larger and clearly visible. The transverse and longitudinal stripes, and trailing phenomenon were less in the graph. The matching rate of three repeat profiles was more than $95 \%$. In general, the three profiles had good repeatability, and could be used for subsequent analysis and mass spectrum identification.

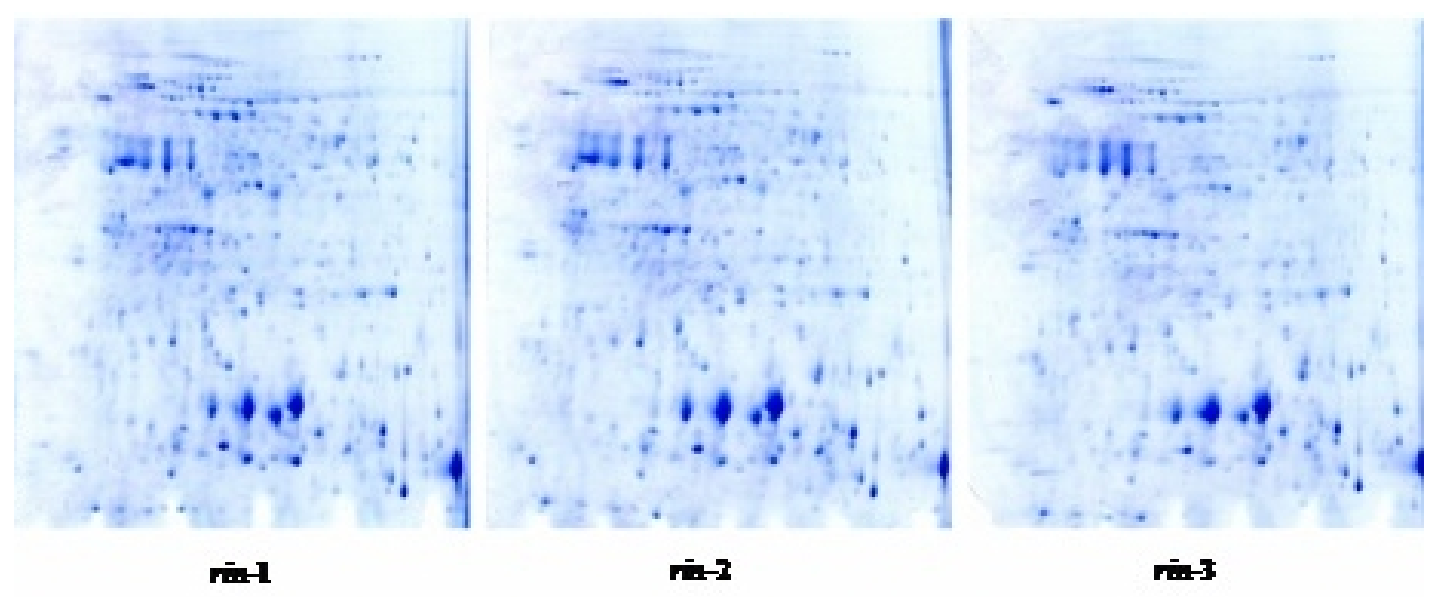

Fig.5 2-DE profile of mutant tomato fruit

\section{Conclusion}

This research had adopted improved TCA-acetone precipitation method to extract the protein of tomato fruit. The lysis buffer contains $9 \mathrm{M}$ protein solubilizer urea, $4 \%$ surface-active agent CHAPS, $0.5 \%$ Triton X-100, 20mM electronating agent DTT. The best loading amount of protein sample and $\mathrm{pH}$ of IPG strips was respectively $500 \mu \mathrm{g}$ and 4-7. The 2-DE profiles under the best condition shows high distinguish ability, which could be used for following analysis.

\section{Acknowledgements}

This work was supported by grants from the National Nature Science Foundation of China (no. 31201662) and Innovative Training Program of Tianjin College Students (no. 201510061073).

\section{References}

[1] G.Nadezha and A.Michail, Proteomics, 2(2002), p. 713-722.

[2] G.Z.Qin, S.P.Tian, Z.L.Chan, et al, Mol Cell Proteomics, 6(2007), p. 425-438.

[3] R.Mariapina, D.Chiara, A.Simona, et al, Proteomics, 6(2006), p. 3718-3791.

[4] J.Vrebalov, D.Ruezinsky, V.Padmanabhan, et al, Science, 296(2002), p. 343-346.

[5] J.H.Liu, B.Y.Xu, J.Zhang, Z.Q.Jin, Hereditas, 32(2010), p. 893-902. 
[6] L.Zhang, Nanjing Agricultural University, (2012), p. 29.

[7] A.Bodzon-Kulakowska, A.Bierczynska-Krzysik, T.Dylag, et al, Chromatog B, 849(2007), p. $1-31$.

[8] E.Katz, M.G.Fon, R.Nakano, et al, Plant Biology, (2006), p. 116.

[9] Y.M.Wang, B.G.Hua and Y.N.Wang, Acta Horticulturae Sinica. 34 (2007), p. 1579 -1584.

[10] C.W.Lu, X.Q.Pan, H.Q.Tian, Y.B.Luo and B.Z.Zhu, Food Science and technology, 35(2010), p. 196-200. 